MARKET WATCH

\section{Upcoming market catalysts in Q1 2020}

Upcoming catalysts in the first quarter of 2020 include US approval decisions for AR101 for peanut allergy and ozanimod for multiple sclerosis.

Astellas and Seattle Genetics are developing enfortumab vedotin for the treatment of urothelial cancer. This antibodydrug conjugate (ADC) targets nectin-4, a cell adhesion molecule overexpressed in solid tumours. In February 2019, long-term results from a phase I trial of enfortumab vedotin in patients with metastatic urothelial cancer showed an encouraging confirmed overall response rate (ORR) of $42 \%$ and a 7.7-month median duration of response (DoR) among responders to treatment. Additional results from the phase II EV-201 study showed a similar ORR of $44 \%$, with complete responses observed in $12 \%$ of treated patients and a median DoR of 7.6 months. Owing to the high unmet need in this area, these positive results significantly increased enfortumab vedotin's likelihood of approval. A biologics license application (BLA) for enfortumab vedotin was subsequently submitted to the FDA in July 2019 for patients who have locally advanced or metastatic urothelial cancer after being treated with a PD1/PDL1 inhibitor or platinum-containing chemotherapy. A decision from the FDA is due by the enfortumab vedotin for urothelial cancer,
Prescription Drug User Act (PDUFA) action date of 15 March 2020. To support global registrations, enfortumab vedotin is also being studied in a pivotal, confirmatory phase III trial known as EV-301.

Aimmune Therapeutics' AR101 is approaching its assigned PDUFA action date in late January 2020. AR101 (which has been given the brand name Palforzia) is an oral capsule formulation immunotherapy of peanut flour and other pharmaceuticalgrade ingredients to treat children and adolescents with a peanut allergy. Aimmune filed a BLA to the FDA in December 2018 based on efficacy data from a phase III study known as PALISADE and long-term safety data from a phase III study called RAMSES. The results are encouraging, with $67 \%$ of patients treated with AR101 tolerating around $600 \mathrm{mg}$ of peanut protein compared with $4 \%$ of patients receiving placebo, and demonstration of a safety profile consistent with the data from PALISADE in the RAMSES study. These headline results outshine data from its late-phase competitor, an epicutaneous patch that delivers a small dose of peanut protein via the skin developed by DBV Technologies and given the brand name Viaskin Peanut, for which a BLA was accepted by the FDA in October 2019. On the basis of AR101's data portfolio, the Allergenic Products Advisory

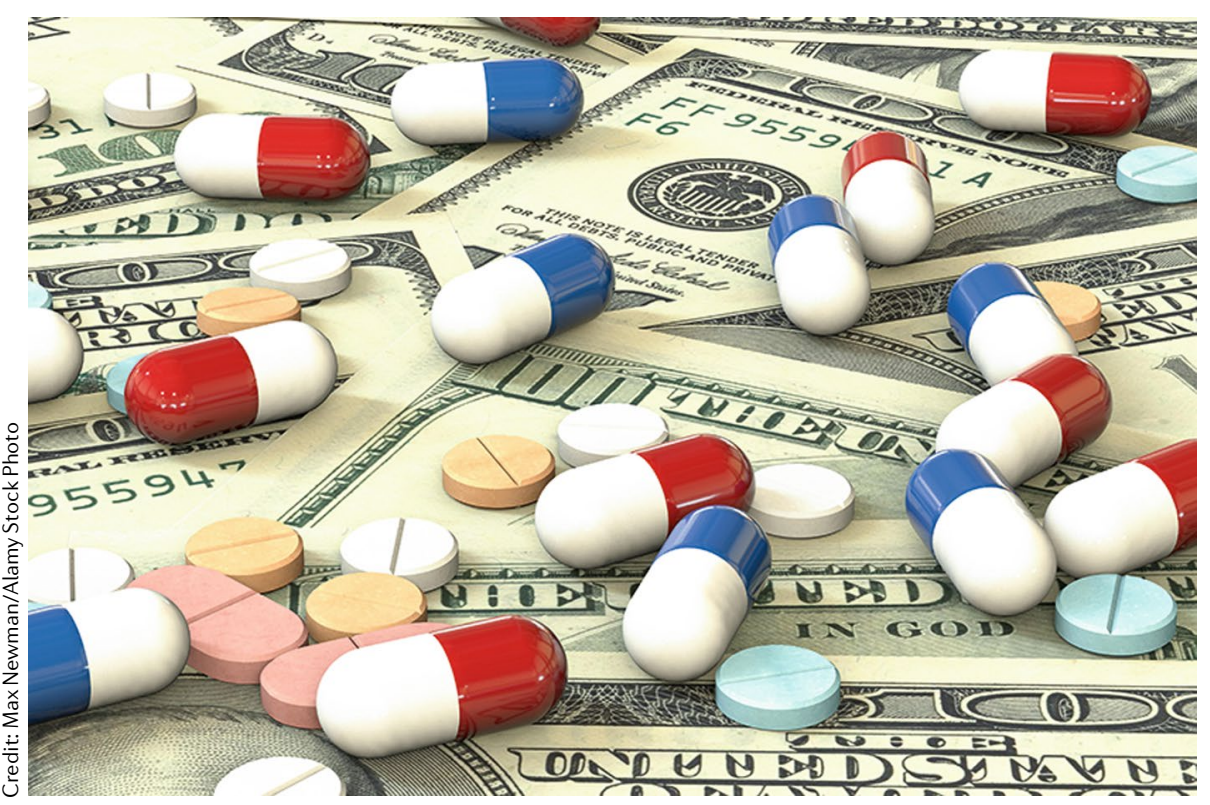

Committee (APAC) convened by the FDA voted in favour of approval for AR101 in September 2019, increasing its already high chance of approval. If approved, AR101 would be the first treatment on the market for peanut allergy. In addition, AR101 has been studied in Europe in a phase III trial known as ARTEMIS. Positive results were also reported, with $58 \%$ of patients who received AR101 tolerating 1,000 mg of peanut protein, reinforcing the efficacy data from PALISADE and adding support for regulatory applications outside the United States.

\section{If approved, AR 101 would} be the first treatment on the market for peanut allergy

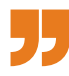

Ozanimod is an oral small-molecule immunomodulator that targets sphingosine 1-phosphate (S1P) 1 and 5 receptors to reduce lymphocyte migration to sites of inflammation, including migration to the central nervous system. This mechanism has shown utility in treating relapsingremitting forms of multiple sclerosis, and the pioneering S1P receptor modulator, fingolimod (Gilenya; Novartis), was approved by the FDA in 2010. Ozanimod, which has greater selectivity for the $\mathrm{S}_{1} \mathrm{P}_{1}$ and $\mathrm{S}_{5} \mathrm{P}_{5}$ receptors than fingolimod, was originally developed by Celgene, until the company's recent acquisition by Bristol-Myers Squibb in November 2019. Ozanimod was studied in two phase III trials, known as SUNBEAM and RADIANCE. In the RADIANCE trial, ozanimod showed a statistically significant reduction of total gadolinium-enhancing (GdE) lesions with a reduction of $86 \%$ compared with placebo. Results from SUNBEAM showed consistent superiority against interferon beta-1a (Avonex; Biogen) in patients with relapsing multiple sclerosis in relapse rate and reduction in MRI lesions at 1 year. Celgene filed a new drug application (NDA) for ozanimod in 2017 but hit a setback in February 2018 when the FDA responded with a refuse-to-file letter asking for more data. Celgene resubmitted the NDA in March 2019 and a new PDUFA target action date of 25 March 2020 is approaching.

$$
\begin{array}{r}
\text { Jenny Tan } \\
\text { Sagient Research Systems, San Diego, } \\
\text { CA, USA. } \\
\text { e-mail: Jenny.Tan@informa.com }
\end{array}
$$$$
\text { https://doi.org/10.1038/d41573-019-00211-8 }
$$ 